\title{
Design of a Computationally Efficient Dynamic System-Level Simulator for Enterprise LTE Femtocell Scenarios
}

\author{
J. M. Ruiz-Avilés, S. Luna-Ramírez, M. Toril, F. Ruiz, I. de la Bandera, P. Muñoz, \\ R. Barco, P. Lázaro, and V. Buenestado \\ Communications Engineering Department, University of Málaga, 29071 Málaga, Spain \\ Correspondence should be addressed to J. M. Ruiz-Avilés, jmruiz@ic.uma.es
}

Received 9 March 2012; Accepted 26 October 2012

Academic Editor: Dharma Agrawal

Copyright (C) 2012 J. M. Ruiz-Avilés et al. This is an open access article distributed under the Creative Commons Attribution License, which permits unrestricted use, distribution, and reproduction in any medium, provided the original work is properly cited.

In the context of Long-Term Evolution (LTE), the next generation mobile telecommunication network, femtocells are lowpower base stations that efficiently provide coverage and capacity indoors. This paper presents a computationally efficient dynamic system-level LTE simulator for enterprise femtocell scenarios. The simulator includes specific mobility and traffic and propagation models for indoor environments. A physical layer abstraction is performed to predict link-layer performance with low computational cost. At link layer, two important functions are included to increase network capacity: Link Adaptation and Dynamic Scheduling. At network layer, other Radio Resource Management functionalities, such as Admission Control and Mobility Management, are also included. The resulting tool can be used to test and validate optimization algorithms in the context of Self-Organizing Networks (SON).

\section{Introduction}

Long-Term Evolution (LTE) is the evolution of the current UMTS mobile communication network. This 3GPP standard is the combination of the all-IP core network known as the evolved packet core (EPC) and the evolved UMTS terrestrial radio access network (E-UTRAN). The key benefits of LTE can be summarized in improved system performance, higher data rates and spectral efficiency, reduced latency and power consumption, enhanced flexibility of spectral usage, and simplified network architecture [1].

In spite of all these advantages, the provision of a high-quality service indoors is still challenging for LTE. Femtocells have been proposed to provide high coverage and capacity indoors without the huge investment required by macrocellular infrastructure [2]. Femtocells are low-power base stations using cellular technology in licensed spectrum bands providing coverage and capacity indoors over an internet-grade backhaul under operator management.

In parallel, Self-Organizing Networks (SON) have arisen as a key topic in next generation networks. The term SON is applied to those networks including self-configuration, self-optimization, and self-healing [3]. In the last years, many SON algorithms have been proposed and tested in macrocellular scenarios (e.g., $[4,5])$. However, few of them have been tested in femtocell scenarios. Note that indoor scenarios have completely different mobility and propagation trends that might cause that SON schemes that have worked well in macrocellular or microcellular environments not to work properly. Moreover, most studies in femtocells have focused on residential scenarios (e.g., [6]). Unlike residential scenarios, enterprise femtocell scenarios have several important differences, namely, (a) enterprise scenarios usually have a three-dimensional structure, where neighboring cells can be located everywhere around the server cell; (b) a different (and probably more intense) mobility pattern; (c) a higher probability of user concentration both in space (e.g., canteen) and time (e.g., coffee break, meeting end); (d) open access instead of closed (i.e., limited) access used in residential femtocells. Open access femtocell refers to those cells allowing any (potentially many) user to transmit. 
Simulation software can be used to aid the design of effective schemes for femtocells. Such tools help to reduce development time and costs while keeping operational risks to a minimum when new algorithms are implemented in the network. With the increasing complexity of mobile networks, these tools become essential in the assessment of SON schemes, where multiple mechanisms and optimization criteria have to be taken into account. This paper describes the design of a dynamic time-driven system-level simulator for voice services in enterprise LTE femtocell networks. A three-dimensional office scenario is built, together with realistic indoor mobility and propagation models. A physical layer abstraction is performed to predict link-layer performance with a low computational cost. Thus, realistic OFDM (Orthogonal Frequency Division Multiplexing) channel realizations with multipath fading propagation conditions have been generated to obtain an accurate value of Signal-to-Interference Ratio (SIR) for each subcarrier. Then, a method is used to aggregate SIR measurements of several OFDM subcarriers into a single scalar value. Subsequently, Block Error Rate (BLER) is estimated from those SIR values, which is used in the Link Adaptation and Dynamic Scheduling algorithms. Additionally, functions for admission control and mobility management are included in the simulator. For simplicity, the tool is only focused on the downlink of E-UTRAN. To show the potential of the simulation tool, a sensitivity analysis of critical handover parameters is performed to identify optimal parameter settings for this specific scenario.

The rest of the paper is organized as follows. Section 2 presents the state of research in femtocell network simulation. Section 3 outlines the main features of LTE radio access technology. Section 4 describes the simulator in detail. Section 5 shows simulation results and Section 6 summarizes the main conclusions of the study.

\section{State of Research}

The design of efficient simulation tools for mobile networks has been widely addressed in the literature. Mobile network simulators can be classified into link-level and system-level simulators. Link-level simulators only consider one link between user and base station and are commonly used to evaluate the influence of the channel on coding performance. System-level simulators consider multiple users and base stations to evaluate the performance of a whole network. The latter can be divided into static and dynamic. Static simulators do not model the movement of users and there is no correlation between consecutive steps. In contrast, dynamic simulators consider causality and time correlation between events in the network. For this reason, they have been used to validate new handover schemes. Dynamic simulators can be further classified into event-driven or time-driven. In an event-driven simulator, routines are only executed based on events (e.g., change of user position, call arrival). Thus, execution time can be reduced when compared to time-driven simulators, where simulation step is fixed. However, the latter can provide accurate network performance statistics that have to be collected periodically.

For LTE, similarly to the evolution of simulation tools in previous radio access technologies, a simple physical layer model was proposed first in order to reduce the complexity of system-level analysis $[7,8]$. Later, a link-level simulator was presented as an appropriate interface to a systemlevel simulator $[9,10]$. Finally, a system-level simulator was proposed as a useful platform for testing planning or optimization techniques [11-13].

Several femtocell simulation tools can also be found in the literature. These differ in the radio access technology, the environment, and the use case for which they were designed.

López-Pérez et al. use a static network simulator in an hybrid scenario (macro- and femtocells) for WiMAX technology to evaluate the performance of different interference avoidance algorithms [14-16]. Their simulations all involve residential scenarios, with both private and open (i.e., public) femtocells.

Claussen et al. simulate UMTS femtocells in a residential scenario $[6,17]$ with static snapshots. [18] evaluates the performance of a femtocell network with identical simulation conditions, but in a generic radio access technology simulation tool. In addition to $[6,17]$, an event-driven simulation is presented in [19] to assess different $\mathrm{HO}$ algorithm configurations. Such a $\mathrm{HO}$ performance analysis is performed over an individual link.

Closer to the simulation tool presented here, [20] implements a 3D scenario with a femtocell network located in a building. Static simulations are carried out in a generic radio access technology to analyze coverage and outage probabilities. Likewise, [21] implements an enterprise femtocell network in 2D for a generic radio access technology in a static simulator, to optimize femtocell transmission powers for a global coverage. Also to evaluate coverage, [22] implements a femtocell network in a 3D scenario (building) where femtocell locations are optimized with static simulations.

Besides these LTE simulation tools, there are other general purpose simulation tools that are not specific for LTE but can be used for this purpose. This is the case of NS3 [23], a discrete event simulator available in the public domain that is very useful for communication network research. Likewise, OMNET++ [24] is a simulation tool commonly used for mobile communication networks. QualNet [25] is also a simulation platform that can predict wireless network and networking device performance, but it is not publicly available.

From this analysis, it can be concluded that simulation tools for femtocell networks are mostly static and/or in residential scenarios. In this work, a 3D enterprise open femtocell scenario is implemented in a dynamic, timedriven, LTE simulation tool.

\section{LTE Basics}

E-UTRAN [26] is composed basically of just one type of node: the base station called evolved Node B (eNB). To reduce network elements, all functions that were included in 
the Radio Network Controller (RNC) in UMTS are located in the eNB in LTE (e.g., radio protocols, mobility management, header compression, and security algorithms). The eNBs are connected by standardized interfaces, called X2, which allow multivendor interoperability. In addition, information such as traffic load can be exchanged between eNBs over the X2 interface.

In LTE, the multiple-access scheme is Orthogonal Frequency-Division Multiplexing (OFDM) in the downlink and Single Carrier Frequency Division Multiple Access (SC-FDMA) in the uplink [27]. These techniques achieve a reduction in the interference, thus increasing network capacity. A Physical Resource Block (PRB), which has a bandwidth resolution of $180 \mathrm{kHz}$, is the minimum amount of frequency resources that can be scheduled for transmission. LTE performs channel-dependent scheduling in both time and frequency, with a resolution of one subframe (1 ms) and one PRB, respectively. The scheduler is a fundamental part of the base station due to its influence over system performance.

LTE defines a set of advanced functions for Radio Resource Management (RRM) in order to achieve an efficient use of the available resources. These functions include radio bearer control, radio admission control, radio mobility control, and scheduling and dynamic allocation of resources. At layer 2, Link Adaptation and Dynamic Packet Scheduling are key features to ensure high spectral efficiency [28] based on user connection quality. On the one hand, Link Adaptation dynamically adjusts the data rate (modulation scheme and channel coding rate) to match the radio channel capacity for each user. The Channel Quality Indicator (CQI) transmitted by the UE, which is an indication of the data rate that can be supported by the channel, is an important input for link adaptation algorithms. On the other hand, Dynamic Scheduling distributes the PRBs among the UEs and the radio bearers of each UE every Transmission Time Interval (TTI) of $1 \mathrm{~ms}$.

At RRM layer 3 [29], Admission Control and Mobility Management are crucial to ensure seamless service as the user moves. Admission Control decides whether the requests for new bearers are granted or rejected, taking into account the available resources in the cell, the QoS requirements for the bearer, the priority levels, and the provided QoS to the active sessions in the cell. Mobility management includes procedures for idle and connected UEs. For both types of procedures, the UE periodically performs not only serving cell quality measurements, but also neighboring cell measurements. In idle mode, cell selection selects a suitable cell to camp on based on radio measurements. In connected mode, handover decides whether the UE should move to another serving cell. The main difference between UMTS and LTE is that in LTE only hard handovers are defined.

\section{Simulation Tool}

This section describes the simulation tool that has been developed in MATLAB R2010a. The following description is divided in four subsection: basic structure, physical layer, link layer, and network layer.

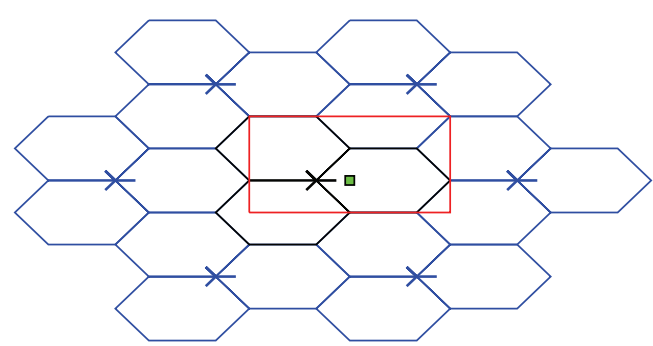

FIGURE 1: Simulation scenario.

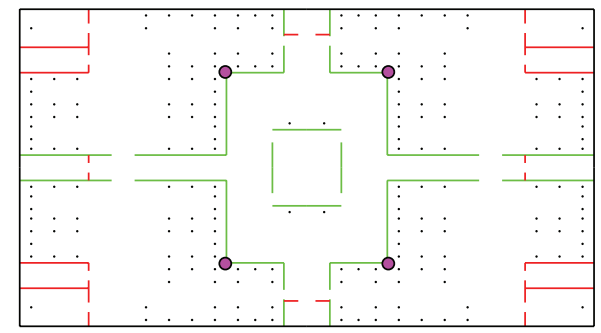

FIgURE 2: A floor diagram.

\subsection{Basic Structure}

4.1.1. Simulation Scenario. Figure 1 shows the simulation scenario of $3 \times 2.6 \mathrm{~km}$, comprising three trisectorized macrocells (black cells) in the same site. To avoid border effects in the simulation, the simulator incorporates the wraparound technique described in [30]. Wrap-around consists of creating replicas of the scenario (blue cells) surrounding the original one. These replicas produce interference in the border of the original scenario, thus avoiding border effects. Only the original scenario is considered when collecting performance statistics.

Inside the coverage area of one macrocell, an office building with dimensions $50 \mathrm{~m} \times 50 \mathrm{~m}$ (green square) has been placed. The number of floors inside the building is configurable ( 5 floors in this work). The floor plan is the same for all floors. Figure 2 shows the layout of one of the floors. Magenta circles reflect femtocells positions, lines are the walls (different colors represent their thickness), and black diamonds are work stations.

4.1.2. Mobility Model. The outdoor mobility model adopted in this work is very simple, since movement only needs to be reflected in a large-scale. Users move with a random direction that not changes and a constant speed of $3 \mathrm{~km} / \mathrm{h}$.

On the other hand, indoor environments are more complex as small user movement may have a strong impact on the signal levels received from base stations. The indoor mobility model developed for the deployment scenario is an extension of the mobility model described in [31]. Users switch between stationary and moving state, but they cannot change floors. A set of location points are defined in the scenario layout so that the user can select them as destination points. Each point has a probability of keeping the user stationary in that location. The time each user spends in 
the stationary state follows a geometric distribution. Points located in office rooms emulate desks, so that they have associated a high probability of keeping the user there. In contrast, points located in corridors have this probability equal to zero, emulating passing locations for the users.

Unlike the approach in [31], where the user switches to the moving state leaving a desk, the destination point can be either desk in the office room or the corridor door. The user speed in the moving state is equal to $1 \mathrm{~km} / \mathrm{h}$. If the corridor door is reached, the user selects the next destination point located in the corridor using a nonuniform distribution. Such a distribution depends on how often the user visits each room. For instance, coffee rooms, meeting rooms, and toilets should be more likely visited. Location points within these rooms are treated the same as office rooms. When the mobile reaches a destination point within a room, it is changed into the stationary state.

The user trajectory is a straight line between source and destination. Thus, there must be direct line of sight with the destination point. For this reason, some auxiliary destination points have been defined within the corridor to avoid crossing walls, as the corridor is not rectangular, as in [31].

Finally, note that the user trajectories are precomputed and stored in a file. Such a piece of information is used as an input during the simulation. Precomputing trajectories, instead of calculating them in real time during simulation, reduces computationally load. An additional benefit is that precomputed trajectories can be reused for different experiments, obtaining repeatability. An example of user trajectories is shown in Figure 3.

4.1.3. Spatial Traffic Distribution. Users can be evenly or unevenly distributed in the scenario. In case of uniform spatial distribution, users are located in any point of the scenario with the same probability. Alternatively, an irregular spatial traffic distribution can be used to reproduce the more realistic situation of user concentration in a canteen, meeting room or coffee room.

In the simulator, it is possible to define the probability with which users start their calls in the coverage area of each cell. This is achieved during the generation of mobility traces by forcing the initial position of each trajectory to be in the selected area.

4.1.4. Traffic Model. The considered service is Voice over IP (VoIP). The VoIP service is defined as a source generating packets of 40 bytes every $20 \mathrm{~ms}$ [32], reaching a bit rate of $16 \mathrm{kbps}$ during a given time (call duration). As will be shown later, the radio resource allocation in the simulator is performed for time intervals of $10 \mathrm{~ms}$. For this reason, the voice service has been implemented as users that transmit packets of 20 bytes every $10 \mathrm{~ms}$.

For this service, it is necessary to determine when a call is dropped, that is, when the service is interrupted. Such an event occurs when a user does not receive packets during a specific time interval. For instance, user packets are not scheduled when the connection quality is below a certain

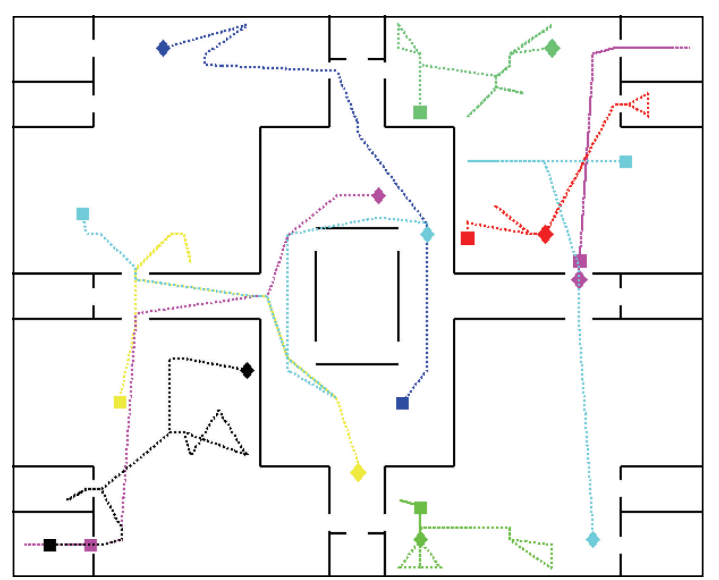

FIGURE 3: User trajectories computed by random waypoint mobility model.

threshold or there are not enough radio resources. In this situation, the call is dropped.

The network traffic load is given by the number of users configured in each simulation. Offered calls are generated following a Poisson distribution with $\lambda=0.42$ calls/user $*$ hour and call duration follows an exponential distribution of mean $100 \mathrm{~s}$.

\subsection{Physical Layer}

4.2.1. Radio Propagation Model. The propagation models used are based on those proposed in the WINNER II project [33]. Such models consider different environments, comprising indoor-indoor, indoor-outdoor, outdoor-indoor, and outdoor-outdoor. All models share the same function structure, including important characteristics as line-ofsight/nonline-of-sight, and wall attenuation and diffraction, defined by

$$
\mathrm{PL}(d B)=A \log (d[m])+B+C \log \left(\frac{f[\mathrm{GHz}]}{5}\right)+X .
$$

Table 1 shows the main parameters of the models for different environments.

When the simulation is running, user location is updated at every iteration, and, consequently, the power received by users from the different BSs has to be updated accordingly. Calculating this data is a time-consuming task. Alternatively, propagation losses can be computed beforehand. Thus, a propagation matrix is computed showing the propagation loss from each BS to each point in the scenario with the aim of improving the computational efficiency. For this purpose, the scenario is divided into a grid of points, each represented by an element in the matrix. Loss values in the matrix are calculated by adding three components: (a) antenna directivity, (b) path loss (PL), and (c) slow fading (SF). Depending on the location of the BS and the mobile station (MS), a different propagation model from those in Table 1 is used to calculate path loss and slow fading. For instance, the signal attenuation from a macrocell to a point inside the 
TABLE 1: Propagation model parameters.

\begin{tabular}{|c|c|c|c|c|}
\hline Scenario & & & Path loss & $\sigma_{\mathrm{SF}}$ \\
\hline \multirow{5}{*}{ Ind2ind } & \multirow{5}{*}{ A1 } & \multirow[t]{2}{*}{ LOS } & $A=18.7, B=46.8, C=20$ & \multirow[t]{5}{*}{6} \\
\hline & & & $A=20, B=46.4, C=20$ & \\
\hline & & \multirow{3}{*}{ NLOS } & $X=5 n_{w}($ Light wall $)$ & \\
\hline & & & $X=12 n_{w}($ Heavy wall $)$ & \\
\hline & & & $\mathrm{FL}=17+4\left(n_{f}-1\right)($ floor att. $)$ & \\
\hline \multirow{4}{*}{ Ind2out } & \multirow{4}{*}{ A2 } & & $\mathrm{PL}=\mathrm{PL}_{b}\left(d_{\mathrm{in}}+d_{\text {out }}\right)+\mathrm{PL}_{t w}+\mathrm{PL}_{\text {in }}\left(d_{\text {in }}\right)$ & 6 \\
\hline & & & $\mathrm{PL}_{b} \rightarrow A=22.7, B=41, C=20$ & \\
\hline & & & $\mathrm{PL}_{t w}=14+15(1-\cos (\theta))$ & \\
\hline & & & $\mathrm{PL}_{\mathrm{in}}=\mathrm{PL}_{A 1}$ & \\
\hline \multirow{3}{*}{ Out2out } & \multirow{3}{*}{$\mathrm{C} 2$} & LOS & $d<d_{\mathrm{BP}} \rightarrow A=26, B=39, C=20$ & 4 \\
\hline & & LUS & $d>d_{\mathrm{BP}} \rightarrow \mathrm{PL}=40 \log (d)+13.47+14 \log \left(h_{\mathrm{BS}}-1\right)-14 \log \left(h_{\mathrm{MS}}-1\right)+6 \log \left(f_{c} / 5\right)$ & 6 \\
\hline & & NLOS & $\mathrm{PL}=\left(44-6.55 \log \left(h_{\mathrm{BS}}\right)\right) \log (d)+34.46+5.83 \log \left(h_{\mathrm{BS}}\right)+23 \log \left(f_{c} / 5\right)$ & 8 \\
\hline Out2ind & $\mathrm{C} 4$ & & $\mathrm{PL}=\mathrm{PL}_{\mathrm{C} 2}\left(d_{\text {in }}+d_{\text {out }}\right)+17.4+0.5 d_{\text {in }}-0.8 h_{\mathrm{MS}}$ & 8 \\
\hline
\end{tabular}

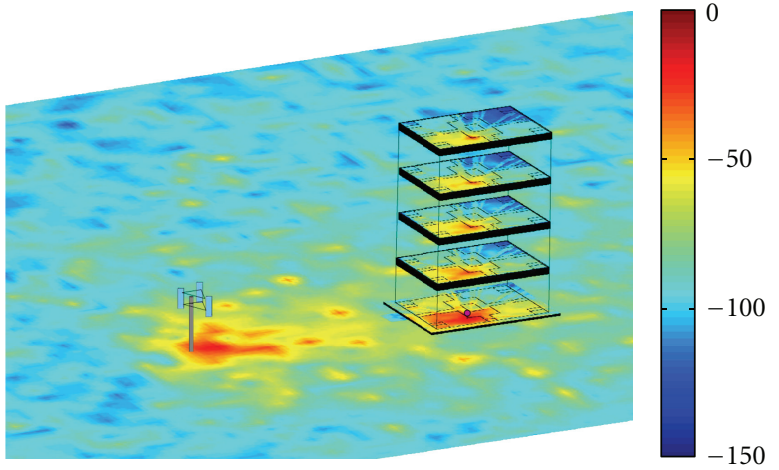

Figure 4: Attenuation grid of a macrocell and a femtocell on the 1st floor $(\mathrm{dB})$.

building is calculated by the $\mathrm{C} 4$ model. For computational efficiency, the A2 model (indoor to outdoor) is implemented only in a 5-meter ring around the building.

Figure 4 shows a colormap with the attenuation in $\mathrm{dB}$ from one sector of the a macrocell and a femtocell located at the ground floor to every point in the scenario (not to scale).

For the definition of the precomputed propagation matrices, the scenario is divided into three grids, one for the outdoor-outdoor environment, other one for the outdoorindoor environment, and the last one for the indoor-indoor and indoor-outdoor environments. The resolution of each grid is $20 \mathrm{~m}, 1 \mathrm{~m}$, and $1 \mathrm{~m}$, respectively. To know the values of the propagation loss a user is experiencing, it is only necessary to read the position of the matrix corresponding to the position occupied by the user in the scenario relative to every base station and then interpolate it with other values of the matrix depending on the relative position in the grid.

4.2.2. Fast-Fading Model. The mobile radio channel can be described as a time-varying linear filter [34]. Therefore, it can be represented in the time domain by its impulse response, $h(\tau, t)$, where $\tau$ stands for delay of each path in the power delay profile, and the amplitude of each path varies with time $t$. When the behavior of the channel is randomly time variant, the above-mentioned channel function becomes a stochastic process. Most practical channels satisfy fairly well the wide-sense stationary uncorrelated scattering (WSSUS) conditions, so their statistical characterization may be accomplished in terms of correlation of the channel function $h$.

As a consequence, a WSSUS channel can be simulated generating the impulse response, $h(\tau, t)$, with stationary variation in time $t$ for each path and no cross-correlation between different values of delay (i.e., generating independent stochastic processes for different paths). Stationarity is achieved by applying Doppler filters to the amplitude time $t$ variation on each path. These filters perform spectrum shaping according to Doppler effect experimented by any radio signal propagating from a transmitter to a moving receiver (or vice versa).

In order to simulate nonconstant speed mobiles (for a femtocell scenario), fading realizations cannot be performed using time $t$ as the independent variable. Alternatively, space variables have to be used so that channel varies according to the current position of the mobile at each iteration of simulation.

Therefore, a fading channel spatial grid has been generated. This grid provides channel responses for every physical position in the simulated scenario, regardless of the speed of the mobiles. In fact, mobiles can stay at a static position for a time interval and then can start moving at any speed. This allows simulation of the femtocell mobility pattern, where mobiles can stop and afterwards go on continuously.

Narrowband fading grid is generated to get a Lord Rayleigh universe of [35]. In other words, following Clarke's model [34], a spatial bidimensional (2D) complex Gaussian variable is filtered by a bidimensional Doppler filter. The bandwidth of 2D Doppler filter can be obtained as a function of spatial grid resolution and wavelength size.

In the considered scenario, where femtocells are located in main rooms, users are often in Line-of-Sight (LOS) with their base stations. This condition introduces the reception 


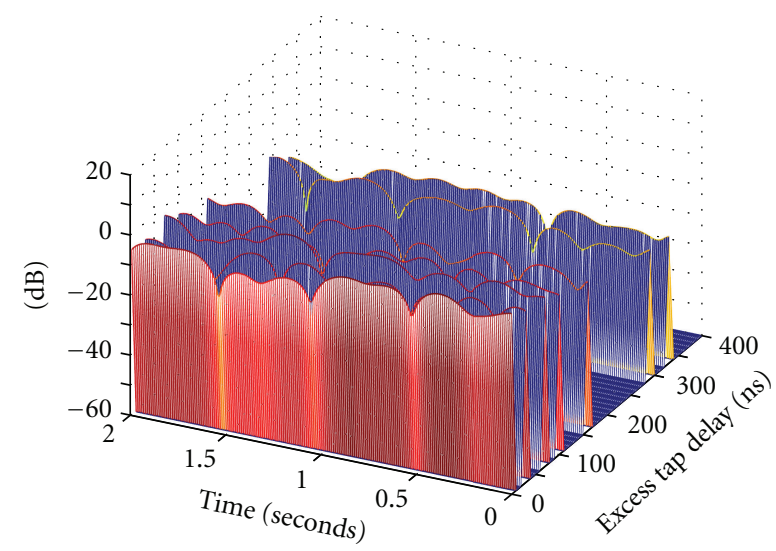

FIGURE 5: Generated bidimensional channel impulse response for Extended Indoor channel model.

of a dominant direct path contribution with considerably higher power than multipath components. Therefore, the amplitude statistics are better modeled by the Rice distribution rather than Rayleigh. The Rice probability density function has a direct-to-multipath power ratio parameter, which, when approximating zero, comes back to the Rayleigh distribution. Rice narrowband grid is simulated by adding the mentioned LOS component to previously generated Rayleigh samples.

Once narrowband channel behavior for each spatial position is obtained, extension to wideband is possible by performing the same procedure for every path in the power delay profile. Thus, different (uncorrelated) Rice grids are generated for each delay in wideband channel scenario. This produces a distance-variant impulse response $h(\tau, d)$ (autocorrelation) of the channel instead of the time-variant impulse response $h(\tau, t)$ described in [34] as one of the four system functions for complete WSSUS (Wide Sense Stationary Uncorrelated Scattering) channel characterization. The only difference is the time to distance ( $t$ to $d$ ) variable change made. A realization of the function for a given mobile route is shown in Figure 5.

Since the simulator requires channel realizations for different frequency bands (corresponding to OFDM subcarriers), the distance-variant impulse response has to be transformed into a distance-variant transfer function $H(f, d)$ at each position, by calculating the Fourier transform of $h(\tau, d)$ with respect to the delay variable. The power delay profile used is an indoor profile that has been extended for an improved modeling of frequency correlation properties [36]. An example of this function for a mobile route can be seen in Figure 6.

The only remaining step is to extend the space variable $d$ of the generated function $H(f, d)$ to a bidimensional $(x, y)$ space variable, obtaining $H(f, x, y)$, a tridimensional function that provides frequency response for each spatial position given its coordinates $x$ and $y$.

To avoid the calculation of fast-fading losses each iteration for each user (which would be very inefficient), these are precomputed before simulation. Three different propagation

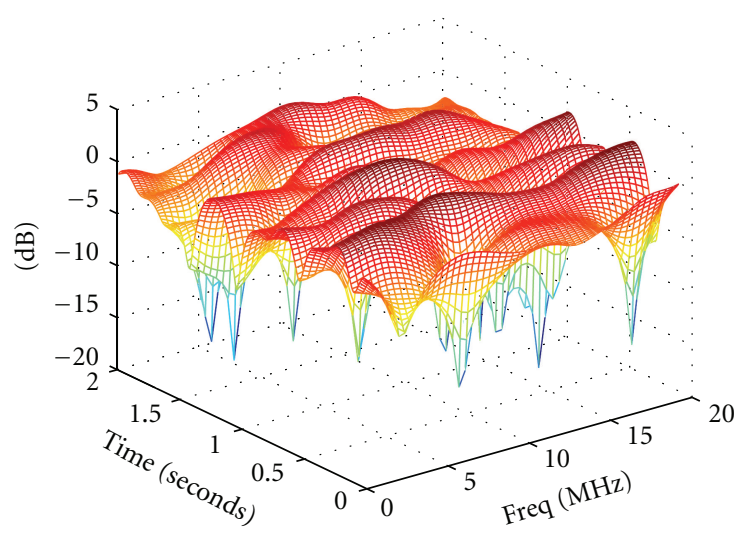

Figure 6: Generated distance-variant transfer function for Extended Indoor channel model.

matrices are constructed, corresponding to outdoor, indoor LOS and indoor NLOS areas, based on standardized profiles (i.e., EPA [36], extended IA [37]). In this case, the grid resolution must be higher than that of slow fading $(15 \mathrm{~cm})$ in order to capture fast fluctuations. The large size of the scenario and the higher resolution avoids building a grid that covers the whole scenario. Instead, a grid of $48 \mathrm{~m} \times 48 \mathrm{~m}$ has been implemented, which is reused to cover the whole scenario.

4.3. Link Layer. In this subsection, four important functionalities of the simulator are described: SIR calculation, Hybrid automatic repeat request scheme, Link adaptation, and Resource scheduling.

4.3.1. SIR Calculation. The SIR is a representative measurement of the link quality that the user is experiencing. To calculate the SIR in the simulator, it is first necessary to calculate the interference experienced by each user. It is assumed that intracell interference is negligible in LTE because the scheduler assigns different frequencies and time slots to each user. Thus, only cochannel intercell interference due to the interfering cells using the same subcarriers is considered. This requires knowing the signal received by each user from all interfering cells. To calculate the interference from each base station to the terminal, the channel response is not taken into account, but only the path loss and slow fading are considered here.

The SIR calculation for a given subcarrier $k, \gamma_{k}$, is computed using the expression proposed in [38]:

$$
\gamma_{k}=P(k) \times \bar{G} \times\left(\frac{N}{N+N_{k}}\right) \times \frac{R_{D}}{N_{S D} / N_{S T}},
$$

where $P(k)$ represents the frequency-selective fading power profile value for the $k$ th subcarrier, $G$ includes the propagation loss, the slow fading, the thermal noise, and the experienced interference, $N$ is the FFT size used in the OFDM signal generation, $N_{p}$ is the length of the cyclic prefix, $R_{D}$ indicates the percentage of maximum total available 
transmission power allocated to the data subcarriers, $N_{S D}$ is the number of data subcarriers per Transmission Time Interval (TTI), and $N_{S T}$ is the number of total useful subcarriers per TTI.

If it is assumed that the multipath fading magnitudes and phases are constant over the observation interval, the frequency selective fading power profile value for the $k$ th subcarrier can be calculated by using the expression:

$$
\begin{array}{r}
P(k)=\left|\sum_{P=1}^{\text {paths }} M_{p} A_{p} e^{j\left[\theta_{p}-2 \Pi f_{k} T_{p}\right]}\right|^{2}, \quad \text { sion: } \\
\bar{G}=\frac{P_{\max }\left(\left(g_{n}(\mathrm{UE}) \times g_{\mathrm{UE}}\right) /\left(\mathrm{PL} \mathrm{UE}, n_{n} \times \mathrm{SH}_{\mathrm{UE}, n}\right)\right)}{P_{\text {noise }}+\sum_{k=1, k \neq n}^{N} P_{\max } \times\left(\left(g_{k}(\mathrm{UE}) \times g_{\mathrm{UE}}\right) /\left(\mathrm{PL}_{\mathrm{UE}, k} \times \mathrm{SH}_{\mathrm{UE}, k}\right)\right)},
\end{array}
$$

where $p$ is the multipath index, $M_{p}$ and $\theta_{p}$ represent the amplitude and the phase values of the multipath fading, respectively, $A_{p}$ is the amplitude value corresponding to the long-term average power for the $p$ th path, $f_{k}$ is the relative frequency offset of the $k$ th subcarrier within the spectrum, and $T_{p}$ is the relative time delay of the $p$ th path. In addition, the fading profile is assumed to be normalized such that $E[P(k)]=1$.

The value of $\bar{G}$ is calculated from the following expres- where $g_{n}(\mathrm{UE})$ is the antenna gain of the serving base station in the direction of the user UE, $g_{\mathrm{UE}}$ is the antenna gain of the user terminal, $P_{\text {noise }}$ is the thermal noise power, $\mathrm{PL}_{\mathrm{UE}, k}$ is the propagation loss between the user and the eNB $k, \mathrm{SH}_{\mathrm{UE}, k}$ is the loss due to slow fading between the user and the eNB $k$ and $N$ is the number of interfering eNBs considered (all the eNBs in the simulator).

A Physical Resource Block (PRB) is the minimum amount of resources that can be scheduled for transmission in LTE. As a PRB comprises 12 subcarriers, it is necessary to translate those SIR values previously calculated for each subcarrier into a single scalar value. This can be made using the Exponential Effective SINR Mapping, which is based on computing the effective SIR by the equation

$$
\operatorname{SIR}_{\mathrm{eff}}=-\beta \operatorname{lm}\left(\frac{1}{N_{u}} \sum_{k=1}^{N_{u}} e^{-\gamma_{k} / \beta}\right),
$$

where $\beta$ is a parameter that depends on the Modulation and Coding Scheme (MCS) used in the PRB [39] assuming that all subcarriers of the PRB have the same modulation, and $N_{u}$ indicates the number of subcarriers used to evaluate the effective SIR. The values of $\beta$ have been chosen so that the block error probability for all the subcarriers is similar to those obtained for the effective SIR in a AWGN channel [40]. The values of $\beta$ for every MCS are shown in Table 2 .

Once the effective SIR has been calculated, the Block Error Rate (BLER) showing the connection quality can be derived. There exist curves that establish the relationship between the values of SIR and BLER defined for an AWGN channel for every modulation and coding rate combination. These curves can also be used to calculate the BLER because intercell interference is equivalent to AWGN as the value of $\beta$ has been selected for this purpose.

4.3.2. Hybrid Automatic Repeat Request Scheme. The Hybrid Automatic Repeat reQuest (H-ARQ) is a function at link level that allows to perform retransmissions directly at physical or MAC layer in LTE. A low-complexity model capable of accurately predicting the H-ARQ gains on the physical layer is derived in [41]. When an H-ARQ retransmission occurs, an improvement of the BLER is expected. The result is that the BLER curves based on AWGN channel model are shifted providing a Signal to Noise Ratio (SNR) gain due to H-ARQ. Hence, the new SIR can be calculated as follows:

$$
\operatorname{SIR}(i)=\operatorname{SIR}+\operatorname{SNR}_{\text {gain }}(i),
$$

where $i$ represents the $i$ th retransmission. The value of $\mathrm{SNR}_{\text {gain }}$, which depends on the redundancy version index $i$ and the given MCS can be derived from a specific table given in [41].

Once the value of BLER has been obtained and taking into account the MCS used in the transmission, it is possible to calculate the throughput value, $T_{i}$, for each user as follows:

$$
T_{i}=\left(1-\operatorname{BLER}\left(\operatorname{SIR}_{i}\right)\right) \times \frac{D_{i}}{\mathrm{TTI}},
$$

where $D_{i}$ is the data block payload in bits [42], which depends on the MCS selected for the user in that time inter$\mathrm{val}$, TTI is the transmission time interval and $\operatorname{BLER}\left(\mathrm{SIR}_{i}\right)$ is the value of BLER obtained from the effective SIR.

4.3.3. Link Adaptation. Before explaining the Link Adaptation function, the 3GPP standardized parameter known as Channel Quality Indicator (CQI) is described. Such an indicator represents the connection quality in a subband of the spectrum. The resolution of the CQI is 4 bits, although a differential CQI value can be transmitted to reduce the CQI signaling overhead. Thus, there is only a subset of possible MCS corresponding to a CQI value [43]. QPSK, 16QAM, and 64QAM modulation schemes may be used in the transmission scheme. In the simulator, the CQI is reported by the user to the base station in each iteration (100 ms).

Based on CQI values, the link adaptation module selects the most appropriate modulation and coding scheme to transmit the information on the physical downlink shared channel (PDSCH) depending on the propagation conditions. The CQI index is used to quantify the link quality for 
TABLE 2: Values of $\beta$ depending on the modulation and coding scheme.

\begin{tabular}{lcc}
\hline Modulation & Cod. & $\beta$ factor \\
\hline QPSK & $1 / 3$ & 1.49 \\
QPSK & $2 / 5$ & 1.53 \\
QPSK & $1 / 2$ & 1.57 \\
QPSK & $3 / 5$ & 1.61 \\
QPSK & $2 / 3$ & 1.69 \\
QPSK & $3 / 4$ & 1.69 \\
QPSK & $4 / 5$ & 1.65 \\
16QAM & $1 / 3$ & 3.36 \\
16QAM & $1 / 2$ & 4.56 \\
16QAM & $2 / 3$ & 6.42 \\
16QAM & $3 / 4$ & 7.33 \\
16QAM & $4 / 5$ & 7.68 \\
64QAM & $1 / 3$ & 9.21 \\
64QAM & $2 / 5$ & 10.81 \\
64QAM & $1 / 2$ & 13.76 \\
64QAM & $3 / 5$ & 17.52 \\
64QAM & $2 / 3$ & 20.57 \\
64QAM & $3 / 4$ & 25.16 \\
64QAM & $4 / 5$ & 28.38 \\
\hline
\end{tabular}

each user and for each subband of the spectrum. If the experienced BLER value is required to be smaller than a specific value given by the service, it is possible to establish a SIR-to-CQI mapping that allows to select the most appropriate MCS for a given SIR value [8]. 3GPP standards define a 5-bit modulation and coding scheme field of the downlink control information to identify a particular MCS. This leads to a greater variety of possible modulation and coding schemes. For simplicity, the developed LTE simulator only includes the same set of MCS given by the CQI index. From the effective SIR value, the index CQI is calculated and the MCS can be determined for the next time interval.

4.3.4. Resource Scheduling. The Resource Scheduling can be decomposed into a time-domain and frequency-domain scheduling. On the one hand, it is necessary to determine which user will transmit at the following time interval. On the other hand, the frequency-domain scheduler selects those subcarriers within the system bandwidth whose channel response is more suitable for the user transmission. For this purpose, the channel response must be estimated for each user and subcarrier in the system bandwidth. Such information is given by the channel realizations generated in the initialization phase of the simulation, assuming a perfect estimation of the channel response. To select the most appropriate frequency subband for the user, the CQI index is used.

The developed simulator includes different strategies for radio resource scheduling. In all of them, the CQI parameter gives the information of the channel quality experienced by each user. Likewise, scheduling is done for each cell at each iteration following the configured strategy
[44]. The following paragraphs describe the four scheduling algorithms implemented in the simulator.

Best Channel Scheduler (BC). In this scheduler, both timedomain and frequency-domain scheduling are done for a more efficient use of resources. At each iteration, all users are sorted based on the quality experienced for each PRB, which is obtained from CQI values. Once the users are sorted, the allocation will proceed until there are no available radio resources or no more users to transmit. The resource allocation is made following the expression

$$
\hat{i}[n]=\arg \max _{i}\left\{r_{i k}[n]\right\}
$$

where $\hat{i}$ is the selected user $i$ and $r_{i k}$ is the estimated achievable throughput for PRB $k$ and user $i$ obtained from the CQI.

This scheduling algorithm maximizes the overall system efficiency because the resource allocation is performed looking for the combinations PRB-user with best channel conditions. The disadvantage of this algorithm is that it harms users with bad channel conditions. Thus, if a user is far from the serving eNB or has a deep fading for prolonged periods of time, it cannot be scheduled and can suffer significant delays.

Round Robin to Best Channel Scheduler (RR-BC). This scheduler uses different strategies for time-domain and frequencydomain scheduling. For time-domain scheduling, the roundrobin method is applied. Thus, users are selected cyclically without taking into account the channel conditions experienced by each of them. Then, each PRB is assigned to the user with a higher potential transmission rate for that PRB (transmission rate is estimated based on the user's CQI value for each PRB).

At each iteration and for each base station, the expressions to be evaluated are

$$
\begin{gathered}
\hat{i}[n+1]=(\hat{i}[n]+1) \bmod N_{u}, \\
\hat{k}[n]=\arg \max _{k}\left\{r_{i k}[n]\right\},
\end{gathered}
$$

where $\hat{i}$ is the selected user, $N_{u}$ is the number of users, and $\hat{k}$ represents the PRB selected. In this case, the goal is to maximize system efficiency, but trying not to harm users with unfavorable channel conditions

Large Delay First to Best Channel Scheduler (LDF-BC). This scheduler is similar to the previous one only differing in time-domain scheduling. In this case, instead of cyclically selecting the users, they are sorted by the time they have been without transmitting. Thus, if for some reason, such as a fading prolonged in time, the user has not been allocated in previous iterations, he will get a higher priority in the current iteration. 
As happened in the previous case, the allocation is carried out at each iteration and for each base station based on the following terms:

$$
\begin{aligned}
& \hat{i}[n]=\arg \max _{i}\left\{W_{i}[n]\right\}, \\
& \hat{k}[n]=\arg \max _{k}\left\{r_{i k}[n]\right\},
\end{aligned}
$$

where $W_{i}[n]$ is the number of iterations without transmitting for user $i$. At the end of each iteration, the value of $W_{i}[n]$ is updated for all the users based on whether they have been allocated or not.

Proportional Fair (PF). The Proportional Fair scheduler is an algorithm similar to best channel, but it tries not to harm users with worse channel conditions. The objective of this algorithm is to find a balance between getting the maximum possible efficiency of the channel and keeping fairness between users. To this end, scheduling is not only based on the potential transmission rate but also takes into account the average transmission rate of the user in previous iterations. The algorithm follows:

$$
\hat{i}[n]=\arg \max _{k}\left\{\frac{r_{i k}[n]}{\bar{r}_{i}}\right\} .
$$

4.4. Network Layer. Network layer functionality comprises those techniques and algorithms giving a global entity to the software tool. Cellular networks are so successful since they care about user mobility giving a global support (coverage, access). While physical and link levels define the propagation and transmission characteristics along the UE-eNB link, network level manages all base stations, terminals and radio resources as a whole.

Main network level functionalities rely on Radio Resource Management (RRM) processes. This section describes the Admission Control (AC), Congestion Control (CC), and Handover ( $\mathrm{HO}$ ) techniques implemented in the simulation tool. It should be pointed out, although scheduling is also usually labeled as an RRM technique, it has already been described in previous sections, since it is located at link level in the simulation tool.

4.4.1. Admission Control. Once an UE decides to start a connection, a first decision is which cell will serve that connection. Such a decision is taken through two main steps:

Minimum Reference Signal Received Power (RSRP). UE collects and sends RSRP to the network from the camping cell and its neighbors. Cells are ordered from higher to lower levels and candidate cells are those fulfilling

$$
\text { RSRP } \geq \text { MinThresholdLEV(i), }
$$

where $\operatorname{RSRP}(i)$ is a wideband measurement meaning the received level for the reference signals in cell $i$, and MinThresholdLEV is the minimum required signal level to be accepted, defined on a cell basis. Finally, the best $i$ cell in the list is initially selected.
Enough Free Resources. The availability of free PRBs in the best cell is then checked. Note that the mobile network does not know how many PRBs the user data connection will require once it is admitted. Signal-level measurements are taken from the reference signals, but radio channel conditions could be quite different for the finally assigned data radio channel (e.g., fast fading, interference). That is the reason why a "worst-case" criterion has been taken to accept UEs. Thus, the UE is finally accepted if

$$
\text { free } P R B \geq \operatorname{maxPRB} \text { (serv), }
$$

where free $\operatorname{PRB}(i)$ is the number of PRBs available in cell $i$, and Max PRB(serv) is the worst-case PRB requirement (i.e., the highest number of PRBs needed to maintain a connection) that a specific type of service, "serv", would demand along the entire connection.

If there are not enough free PRBs, the next candidate cell in the list is checked. A user connection is blocked when no cell fulfills 14 and 15.

4.4.2. Handover. The $\mathrm{HO}$ algorithm is the main functionality to manage the connected user mobility. $\mathrm{HO}$ algorithms are vendor specific. The following paragraphs describe three classical handover algorithms proposed for LTE and implemented in the simulator:

Quality Handover (QualHO). A QualHO is triggered when

$$
\begin{gathered}
\operatorname{RSRQ}(i) \leq \operatorname{RSRQ}(j) \quad \text { for } \operatorname{TTT}^{\text {Qual }} s, \\
\operatorname{RSRP}(j)-\operatorname{RSRP}(i) \geq \operatorname{Margin}_{\text {Qual }}(i, j),
\end{gathered}
$$

where RSRQ is the Reference Signal Received Quality, usually measured by the Signal to Interference and Noise Ratio (SINR) for the reference signals, RSRP is the Reference Signal Received Power, TTT ${ }^{\text {Qual }}$ is the Time-to-Trigger value,

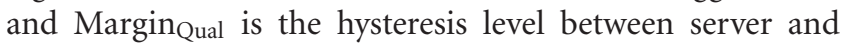
adjacent cells ( $i$ and $j$, resp.). This QualHO aims to reallocate connections which are experiencing a bad quality connection

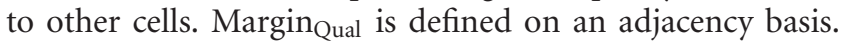
For monitoring purposes, a QualHO is classified as an Interference $\mathrm{HO}$ (IntHO) if

$$
\operatorname{RSRP}(i) \geq \operatorname{RxLEV}_{\text {threshold }}^{\text {Interf }}(i) \quad \text { for TTT } s,
$$

that is, the UE has a high signal level but low SINR figures.

Minimum Level Handover (LevHO). A LevHO is triggered when

$$
\begin{gathered}
\operatorname{RSRP}(i) \leq \operatorname{MinRxLEV}_{\mathrm{LevHO}}(i), \\
\operatorname{RSRP}(j)-\operatorname{RSRP}(i) \geq \operatorname{Margin}_{\text {Lev }}(i, j),
\end{gathered}
$$

where MinRxLEVLevHO is a minimum signal level threshold. LevHO aims to re-allocate connections experiencing a very low signal level (e.g., when the UE is getting out of coverage area). LevHO is considered an "urgent" $\mathrm{HO}$ and must be triggered as soon as possible. Thus, no Time-toTrigger parameter has been considered. 
TABLe 3: Simulation parameters.

\begin{tabular}{|c|c|c|}
\hline Time resolution & $100 \mathrm{~ms}$ & \\
\hline \multirow{4}{*}{ Propagation models } & Indoor-indoor & Winner II A1 \\
\hline & Indoor-outdoor & Winner II A2 \\
\hline & Outdoor-outdoor & Winner II C2 \\
\hline & Outdoor-indoor & Winner II C4 \\
\hline \multirow{5}{*}{ BS model } & \multirow{2}{*}{ PIRE } & $\mathrm{BS}_{\text {femto }}=13 \mathrm{dBm}$ \\
\hline & & $\mathrm{BS}_{\text {macro }}=43 \mathrm{dBm}$ \\
\hline & \multirow{2}{*}{ Directivity } & Femto: omnidirectional \\
\hline & & Macro: trisectorial \\
\hline & Access & Macro/femto: open access \\
\hline \multirow{2}{*}{ MS model } & Noise figure & $9 \mathrm{~dB}$ \\
\hline & Noise spectral density & $-174 \mathrm{dBm} / \mathrm{Hz}$ \\
\hline \multirow{4}{*}{ Traffic model } & \multirow{2}{*}{ Calls } & Poisson \\
\hline & & (avg. 0.42 calls/(user $*$ hour) ) \\
\hline & Duration & Exponential (avg. $180 \mathrm{sec}$ ) \\
\hline & Spatial distribution & Uniform \\
\hline \multirow{2}{*}{ Mobility model } & Outdoor & $3 \mathrm{~km} / \mathrm{h}$, random direction, and wrap-around \\
\hline & Indoor & Random waypoint \\
\hline Service model & Voice over IP & $16 \mathrm{kbps}$ \\
\hline \multirow{6}{*}{ RRM model } & 6 PRBs $(1.4 \mathrm{MHz})$ & \\
\hline & Cell Reselection & $\mathrm{C} 1-\mathrm{C} 2$ \\
\hline & Access control & DR \\
\hline & Handover & PBGT, Qual, Lev \\
\hline & \multirow{2}{*}{ Scheduler: RR-BC } & Time: round-robin (RR) \\
\hline & & Freq.: best channel (BC) \\
\hline
\end{tabular}

Power Budget Handover (PBGT_HO). A PBGT_HO is triggered when

$$
\operatorname{RSRP}(j)-\operatorname{RSRP}(i) \geq \operatorname{Margin}_{\mathrm{Lev}}(i, j) .
$$

In this case, there is no first condition to be fulfilled. Equation (19) is only evaluated every $N^{\text {PBGT }}$ seconds. PBGT_HO is not considered an urgent $\mathrm{HO}$, but an optimization algorithm. At the end of a PBGT_HO process, the UE should be connected to the best cell in terms of signal level (provided that Margin ${ }_{\mathrm{PBGT}}$ is positive).

\section{Performance Analysis}

This section shows an example of how the simulation tool can be used to evaluate the performance of an enterprise femtocell network. In particular, the following paragraphs analyze the tradeoff between connection quality and signaling load when tuning $\mathrm{HO}$ parameters in an enterprise femtocell scenario. From the presented results, optimal network parameter settings can be identified for this specific scenario. For clarity, the simulation set-up is described first and simulations results are then described.

5.1. Simulation Setup. Table 3 summarizes the main simulation parameters. As described in Section 4.1.1-A1 simulation scenario includes an office building with femtocells. This building comprises 5 floors with 4 femtocells each. For simplicity, the floor plan and femtocell positions are the same in all floors. This building is located in a larger macrocellular area, covered by 3 cosited trisectorized cells,. Figures 1 and 2 show BS locations. Every BS includes 6 PRBs. Service model is Voice-over-IP. Network traffic load is very nonuniformly distributed, with a higher user concentration in the middle floor. All cell adjacencies are considered for HO purposes. Dropped calls are enabled (i.e., a call is dropped if it does not receive any radio resource for $1 \mathrm{~s}$ due to lack of resources or bad radio link conditions).

A sensitivity analysis is performed to check the influence of tuning $\mathrm{HO}$ parameters, namely $\mathrm{HO}$ margin (HOM) and Time-to-Trigger (TTT). HOM refers to all hysteresis values defined in (15), (18) and (19), so all hysteresis values from different $\mathrm{HO}$ algorithms are identically modified, while TTT refers to all trigger values defined in (14) and (16). This HOM and TTT considered parameters are those ensuring the quality of the HO process, [1]. During the experiments, all possible combinations of the previous parameters are tested. Each parameter setting is simulated for 72000 iterations of 100 ms (i.e., 2 hours of network time) to obtain network performance statistics.

The considered key performance indicators (KPIs) are the dropped call ratio (DCR), as a measure of network connection quality, and the handover ratio (HOR) (i.e., the average number of HOs per carried call) as a measure of 
network signaling load. To ease the analysis, a single figure of merit (FoM) is computed by aggregating both measures as follows:

$$
\mathrm{FoM}=\omega_{1} \times\left(\frac{\mathrm{DCR}}{\mathrm{DCR}_{t}}\right)^{e}+\omega_{2} \times\left(\frac{\mathrm{HOR}}{\mathrm{HOR}_{t}}\right)^{e},
$$

where DCR and HOR are the performance figures of the configuration, $\mathrm{DCR}_{t}$ and $\mathrm{HOR}_{t}$ are performance target values defined by the operator, $\omega_{1}$ and $\omega_{2}$ are relative weights showing operator preference between connection quality and signaling, and $e$ is a constant to penalize the nonfulfillment of objectives. Hereafter, $\omega_{1}=\omega_{2}=0.5, \mathrm{DCR}_{t}=0.02$ and $\mathrm{HOR}_{t}=0.25$.

Three different situations of average network load (i.e., $25 \%, 50 \%$, and $75 \%$ ) are tested to check whether the conclusions are the same with different values of traffic demand. The overall traffic demand is controlled by changing the number of users in the scenario. To reduce stochastic fluctuations in the indicators for different loops, the number of calls starting in each simulation step, as well as their duration and trajectory, are precomputed off-line and shared by all tested parameter settings. Moreover, small confidence intervals (i.e., below $0.1 \%$ ) for performance measures are observed even with low traffic values. Hence, only average values for indicators will be shown hereafter.

5.2. Simulation Results. A first experiment considers an average network load of $50 \%$. Figures $7(a)-7(\mathrm{c})$ presents the optimization surfaces of DCR, HOR and FoM for different combinations of HOM and TTT. In Figure 7(a), it is observed that the higher the margin value, the larger DCR. It is also clear that the sensitivity to changes in TTT is much lower. Nonetheless, for low HOM values, the larger the TTT, the larger DCR. Both results are logical since increasing either HOM or TTT causes a delay in the HO, which most often leads to a temporary deterioration of connection quality. Figure 7(b) confirms that raising $\mathrm{HO}$ constraints by increasing HOM and TTT leads to less handovers and, hence, a lower HOR. Similar results have been reported for macrocells scenarios [5].

From the previous results, it can be concluded that there is a tradeoff between DCR and HOR when setting HOM and TTT values. Large values of these parameters lead to high DCR and low HOR, whereas small values lead to low DCR but high HOR. Such a tradeoff is observed in the FoM optimization surface, shown in Figure $7(\mathrm{c})$. A deep valley with the lowest (best) FoM values best is observed for medium HOMs (i.e., $3 \mathrm{dBs}$ ) and low TTTs (i.e., $100 \mathrm{~ms}$ ), or low HOMs (i.e., close to zero) and large TTTs (i.e., above $700 \mathrm{~ms}$ ). In particular, the optimal configuration is $\mathrm{HOM}=2 \mathrm{~dB}$ and TTT $=800 \mathrm{~ms}$, for which a FoM of 3.046 is obtained. HOM values are coherent with the value of the slow fading constant in the scenario, ranging from 0 to $8 \mathrm{~dB}$. It should be pointed out that, although large FoM differences (of up to a 4.69) are observed between configurations, the gradient of the surface near the optimum has a small magnitude. Thus, tuning is easier around the optimal area, and the loss due to the discrete nature of TTT and $\mathrm{HOM}$ values is negligible.

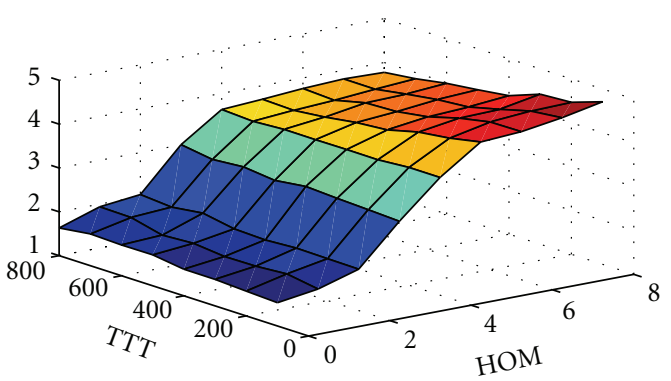

(a) Dropped call ratio

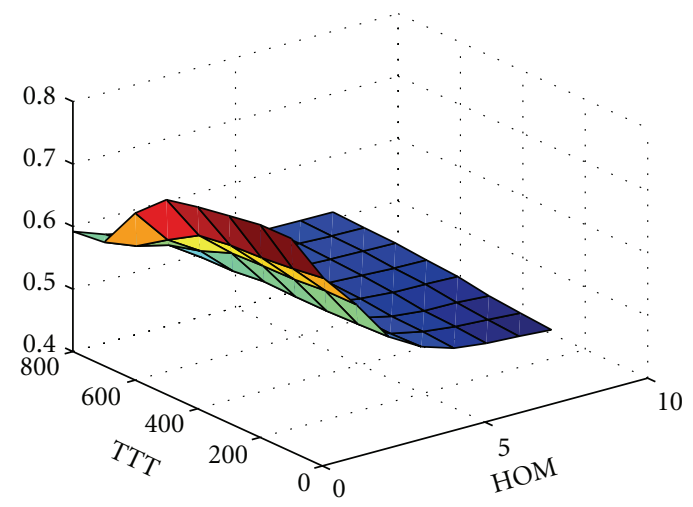

(b) Average number of HOs per call

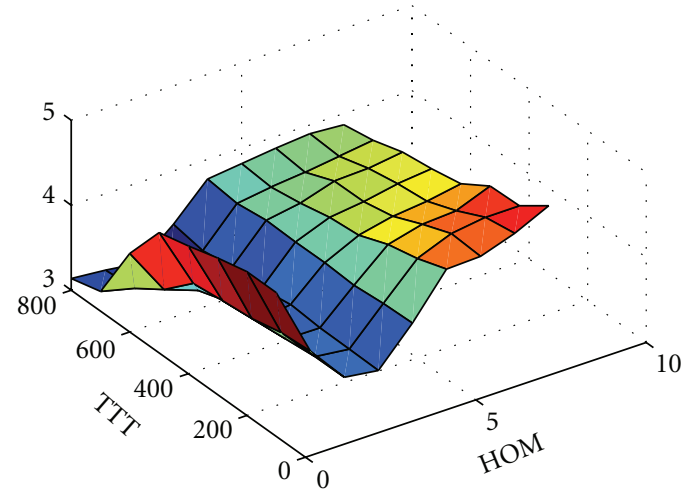

(c) Figure of merit

Figure 7: Results for an average load of 50\%.

A second experiment evaluates the impact of traffic demand on optimal parameter settings. Figures 8 and 9 present the DCR, HOR, and FoM for an average network load of $25 \%$ and $75 \%$, respectively. Figure 8 shows that, for low traffic load values, DCR becomes negligible. A closer analysis shows that this is mainly due to a lower cochannel interference and a lower probability of rejecting an urgent HO due to lack of radio resources. Thus, FoM is governed by HOR and, therefore, the best combinations are those leading to a lower HOR (i.e., larger HOM and smaller TTT values). More specifically, the optimal settings are $\mathrm{HOM}=$ $8 \mathrm{~dB}$ and TTT $=200 \mathrm{~ms}$. In contrast, for high traffic load values, Figure 9, DCR increases significantly above the target value, $\mathrm{DCR}_{t}$ (up to 7 times higher). In these conditions, 


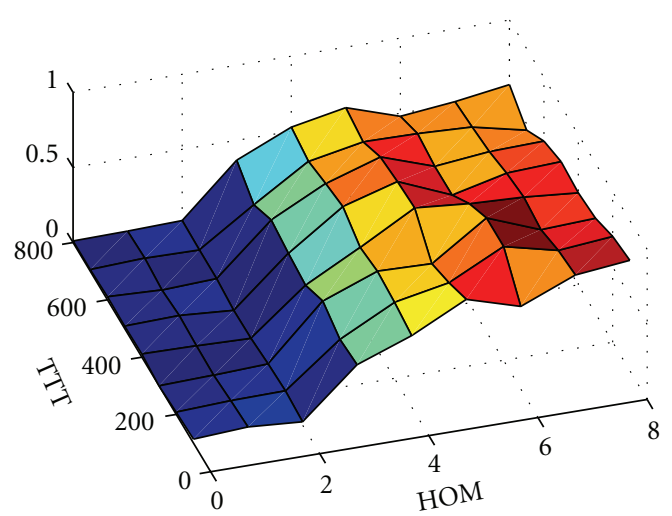

(a) Dropped call ratio

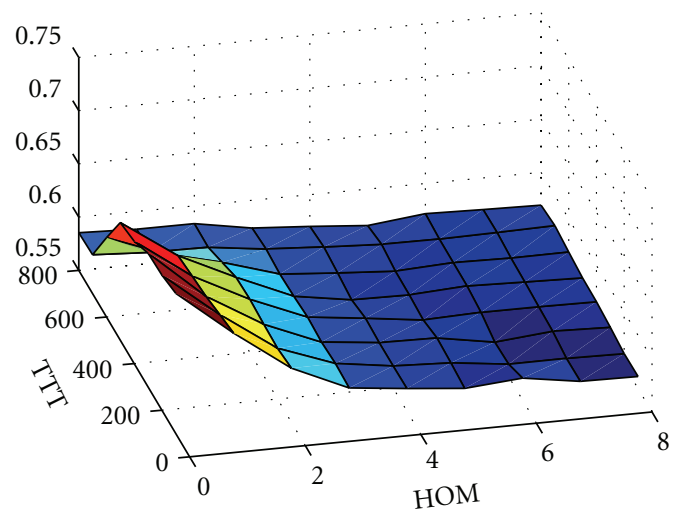

(b) Average number of HOs per call

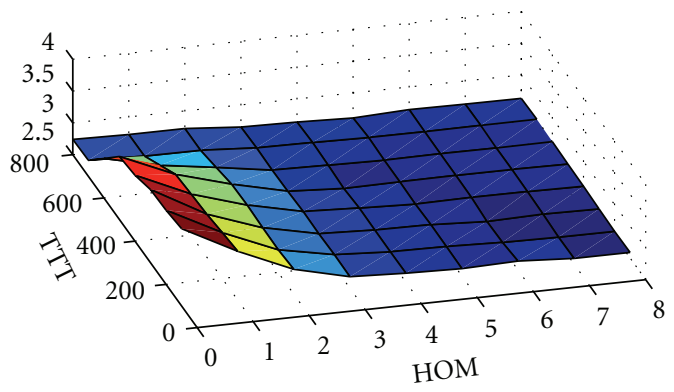

(c) Figure of merit

FIGURE 8: Results for an average load of 25\%.

FoM is mainly governed by DCR and, therefore, the best combinations are those leading to a lower DCR, keeping the HOR not too high (specifically, HOM $=0 \mathrm{~dB}$ and TTT $=$ $800 \mathrm{~ms}$ ).

From the previous results, it can be concluded that the difference between optimal parameter settings can be significant between cases (i.e., $\mathrm{HOM}=8 \mathrm{~dB}$ and TTT $=$ $200 \mathrm{~ms}$ for $25 \%$ load and HOM $=0 \mathrm{~dB}$ and $\mathrm{TTT}=$ $800 \mathrm{~ms}$ for $75 \%$ load). Likewise, selecting the wrong settings can lead to severe impairment of network performance. For instance, fixing $\mathrm{HOM}=8 \mathrm{~dB}$ and TTT $=200 \mathrm{~ms}$ (i.e., the optimal configuration for $25 \%$ load) in the $75 \%$ load scenario means deteriorating FoM by a factor of 2 compared to the optimum. A deeper analysis shows that the most robust combination in the considered enterprise

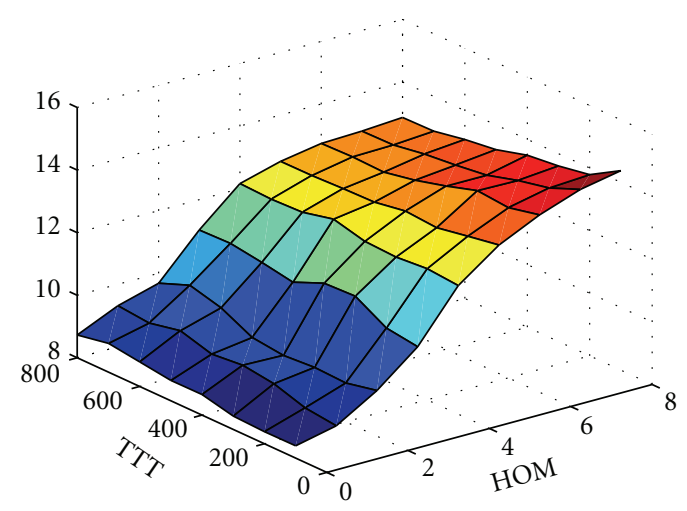

(a) Dropped call ratio

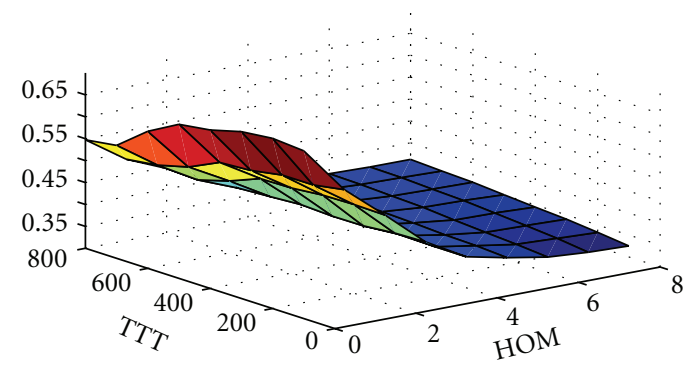

(b) Average number of HOs per call

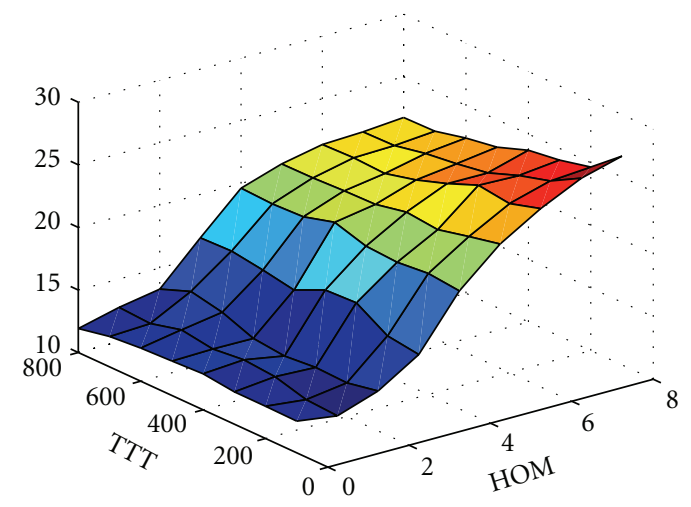

(c) Figure of merit

Figure 9: Results for an average load of $75 \%$. femtocell environment is $\mathrm{HOM}=2 \mathrm{~dB}$ and TTT $=800 \mathrm{~ms}$. With such settings, FoM ranges between 2.72 and 13.31. Nonetheless, results suggest that the best performance can only be obtained by dynamically adapting network parameter settings to traffic load fluctuations.

Finally, it is worth mentioning that simulation times are kept within reasonable limits. Note that deriving each point in the optimization surfaces in Figures 7, 8, and 9, needs 72000 simulation steps to be simulated. Obviously, computational load depends on the number of active users in the scenario, which is modified to model different average traffic load values in the system. In particular, in the worst case (e.g., $75 \%$ case), the simulation time for the referred 72000 steps ( $2 \mathrm{~h}$ of network time) is below $2 \mathrm{~h}$, which is more than acceptable. 


\section{Conclusions}

In this paper, a computationally efficient dynamic systemlevel LTE simulator for enterprise femtocell scenarios has been presented. Unlike similar tools that can be found in the literature, the simulator includes a 3D office scenario with realistic mobility and propagation models. The tool has been designed to reduce computations in real time by precomputing propagation matrices and mobility traces. Likewise, a physical layer abstraction has been performed to predict linklayer performance with a low computational cost. In spite of its high computational efficiency, at link and network layer, the simulator includes most important features offered by LTE vendor equipment. Thus, the resulting tool is well suited for the validation of self-tuning algorithms, where multiple optimization loops have to be executed.

To show the value of the simulator, a sensitivity analysis has been performed to show the impact of the Handover Time-to-Trigger and margin parameters in this specific scenario. Results have shown that the optimal settings for these parameters depend on traffic load conditions. Such a result suggests that the best performance can only be obtained by dynamically adapting network parameter settings to traffic load fluctuations.

\section{Acknowledgments}

This work has been funded by the Spanish Ministry of Science and Innovation (Grant TEC2009-13413) and Junta de Andalucía (Grant TIC-4052).

\section{References}

[1] S. Sesia, M. Baker, and I. Toufik LTE, The UMTS Long Term Evolution: From Theory to Practice, John Wiley \& Sons, New York, NY, USA, 2009.

[2] V. Chandrasekhar, J. G. Andrews, and A. Gatherer, "Femtocell networks: a survey," IEEE Communications Magazine, vol. 46, no. 9, pp. 59-67, 2008.

[3] 3GPP, "Self-Organizing Networks (SON), Concepts and requirements," in TS 32. 500.

[4] A. Lobinger, S. Stefanski, T. Jansen, and I. Balan, "Load balancing in downlink LTE self-optimizing networks," in Proceedings of the IEEE 71st Vehicular Technology Conference (VTC'10), pp. 1-5, Taipei City, Taiwan, May 2010.

[5] P. Muñoz, R. Barco, I. de la Bandera, M. Toril, and S. Luna-Ramírez, "Optimization of a fuzzy logic controller for handover-based load balancing," in Proceedings of the IEEE 73rd IEEE Vehicular Technology Conference (VTC'11), pp. 1-5, Budapest, Hungary, May 2011.

[6] H. Claussen, "Performance of macro- and co-channel femtocells in a hierarchical cell structure," in Proceedings of the 18th Annual IEEE International Symposium on Personal, Indoor and Mobile Radio Communications (PIMRC'07), pp. 1-5, Athens, Greece, September 2007.

[7] J. Wu, Z. Yin, J. Zhang, and W. Heng, "Physical layer abstraction algorithms research for 802.11n and LTE downlink," in Proceedings of the International Symposium on Signals, Systems and Electronics (ISSSE'10), pp. 1-4, Nanjing, China, September 2010.
[8] C. Mehlfhrer, M. Wrulich, J. C. Ikuno, D. Bosanska, and M. Rupp, "Simulating the long term evolution physical layer," in Proceedings of the European Signal Processing Conference (EUSIPCO'09), August 2009.

[9] J. J. Sánchez, G. Gómez, D. Morales-Jiménez, and J. T. Entrambasaguas, "Performance evaluation of OFDMA wireless systems using WM-SIM platform," in Proceedings of the ACM International Workshop on Mobility Management and Wireless Access (MobiWAC'06), pp. 131-134, October 2006.

[10] J. Olmos, A. Serra, S. Ruiz, M. García-Lozano, and D. Gonzalez, "Link level simulator for LTE Downlink," in Proceedings of the 7th European Meeting COST-2100, February 2009.

[11] J. C. Ikuno, M. Wrulich, and M. Rupp, "System level simulation of LTE networks," in Proceedings of the IEEE 71st Vehicular Technology Conference (VTC'10-Spring), pp. 1-5, Taipei City, Taiwan, May 2010.

[12] M. Simsek, T. Akbudak, B. Zhao, and A. Czylwik, "An LTEfemtocell dynamic system level simulator," in Proceedings of the International ITG Workshop on Smart Antennas (WSA'10), pp. 66-71, Bremen, Germany, February 2010.

[13] G. Piro, L. A. Grieco, G. Boggia, F. Capozzi, and P. Camarda, "Simulating LTE cellular systems: an open-source framework," IEEE Transactions on Vehicular Technology, vol. 60, no. 2, pp. 498-513, 2011.

[14] D. López-Pérez, A. Valcarce, G. de la Roche, E. Liu, and J. Zhang, "Access methods to WiMAX femtocells: a downlink system-level case study," in Proceedings of the 11th IEEE Singapore International Conference on Communication Systems (ICCS'08), pp. 1657-1662, Guangzhou, China, November 2008.

[15] D. López-Pérez, G. de la Roche, A. Valcarce, A. Jüttner, and J. Zhang, "Interference avoidance and dynamic frequency planning for WiMAX femtocells networks," in Proceedings of the 11th IEEE Singapore International Conference on Communication Systems (ICCS'08), pp. 1579-1584, Guangzhou, China, November 2008.

[16] D. López-Pérez, A. Valcarce, G. de la Roche, and J. Zhang, "OFDMA femtocells: a roadmap on interference avoidance," IEEE Communications Magazine, vol. 47, no. 9, pp. 41-48, 2009.

[17] L. T. W. Ho and H. Claussen, "Effects of user-deployed, cochannel femtocells on the call drop probability in a residential scenario," in Proceedings of the 18th Annual IEEE International Symposium on Personal, Indoor and Mobile Radio Communications (PIMRC'07), pp. 1-5, Athens, Greece, September 2007.

[18] O. Namgeol, S. W. Han, and H. Kim, "System capacity and coverage analysis of femtocell networks," in Proceedings of the IEEE Wireless Communications and Networking Conference 2010 (WCNC'10), pp. 1-5, Sydney, Australia, April 2010.

[19] H. Claussen, L. T. W. Ho, and L. G. Samuel, "Selfoptimization of coverage for femtocell deployments," in Proceedings of the 7th Annual Wireless Telecommunications Symposium (WTS'08), pp. 278-285, Pomona, Calif, USA, April 2008.

[20] P. Pirinen, "Co-channel co-existence study of outdoor macrocell and indoor femtocell users," in Proceedings of the European Wireless Conference (EW'10), pp. 207-213, Lucca, Italy, April 2010.

[21] I. Ashraf, H. Claussen, and L. T. W. Ho, "Distributed radio coverage optimization in enterprise femtocell networks," in Proceedings of the IEEE International Conference on Communications (ICC'10), pp. 1-6, Cape Town, South Africa, May 2010.

[22] K. Han, Y. Choi, D. Kim, M. Na, S. Choi, and K. Han, "Optimization of femtocell network configuration under 
interference constraints," in Proceedings of the 7th International Symposium on Wireless Networks Modeling and Optimization in Mobile, Ad Hoc, and Wireless Networks (WiOPT'09), pp. 17, Seoul, South Korea, June 2009.

[23] http://www.nsnam.org/.

[24] http://www.omnetpp.org/.

[25] http://www.scalable-networks.com.

[26] 3GPP, "Evolved universal terrestrial radio access (EUTRA) and evolved universal terrestrial radio access network (EUTRAN); overall description," in TS 36.300 .

[27] 3GPP, "Evolved universal terrestrial radio access (EUTRA), LTE physical layer; general description," in TS 36.201.

[28] H. Holma and A. Toskala, LTE for UMTS OFDMA and SCFDMA Based Radio Access, John Wiley \& Sons, New York, NY, USA, 2009.

[29] 3GPP, "Evolved universal terrestrial radio access (EUTRA), requirements for support of radio resource management," in TS 36.133.

[30] T. Hytone, "Optimal wrap-around network simulation," Tech. Rep., Helsinki University of Technology, Institute of Mathematics, 2001.

[31] ETSI, "Universal mobile telecommunications system (UMTS), selection procedures for the choice of radio transmission technologies of the UMTS," in TR 101 112, V3.2.0 (1998-04).

[32] NGMN, "NGMN radio access performance evaluation methodology," in Version 1. 0, 2008, http://www.ngmn.org/.

[33] "D1. 1. 2. WINNER II channel models. part II. radio channel measurement and analysis results. v1.0," Tech. Rep., WINNER II IST Project, 2007.

[34] J. Parsons, The Mobile Radio Propagation Channel, PenTech, 1992.

[35] W. Jakes, Microwave Mobile Communications, John Wiley \& Sons, New York, NY, USA, 1974.

[36] T. Sorensen, P. Mogensen, and F. Frederiksen, "Extension of the ITU channel models for wideband (OFDM) systems," in Proceedings of the IEEE 62nd Vehicular Technology Conference (VTC'05), pp. 392-396, September 2005.

[37] 3GPP, "Evolved universal terrestrial radio access (E-UTRA), user equipment (UE) radio transmission and reception," in TS 36.101.

[38] 3GPP, "Feasibility study for orthogonal frequency division multiplexing (OFDM) for UTRAN enhancement," in TS 25.892.

[39] 3GPP, "System analysis of the impact of CQI reporting period in DL SIMO OFDMA," in R1-061506.

[40] E. Tuomaala and H. Wang, "Effective SINR approach of link to system mapping in OFDM/multi-carrier mobile network," in Proceedings of the IEE Mobility Conference 2005: The 2nd International Conference on Mobile Technology, Applications and Systems, p. 140, Guangzhou, China, November 2005.

[41] J. C. Ikuno, M. Wrulich, and M. Rupp, "Performance and Modeling of LTE H-ARQ," in Proceedings of the International ITG Workshop on Smart Antennas (WSA'09), 2009.

[42] 3GPP, "OFDM-HSDPA system level simulator calibration," in R1-040500.

[43] 3GPP, "E-UTRA, UE conformance specification, radio transmission and reception; part 1: conformance testing," in TS 36.521 .

[44] J. T. Entrambasaguas, M. C. Aguayo-Torres, G. Gómez, and J. F. Paris, "Multiuser capacity and fairness evaluation of channel/QoS-aware multiplexing algorithms," IEEE Network, vol. 21, no. 3, pp. 24-30, 2007. 

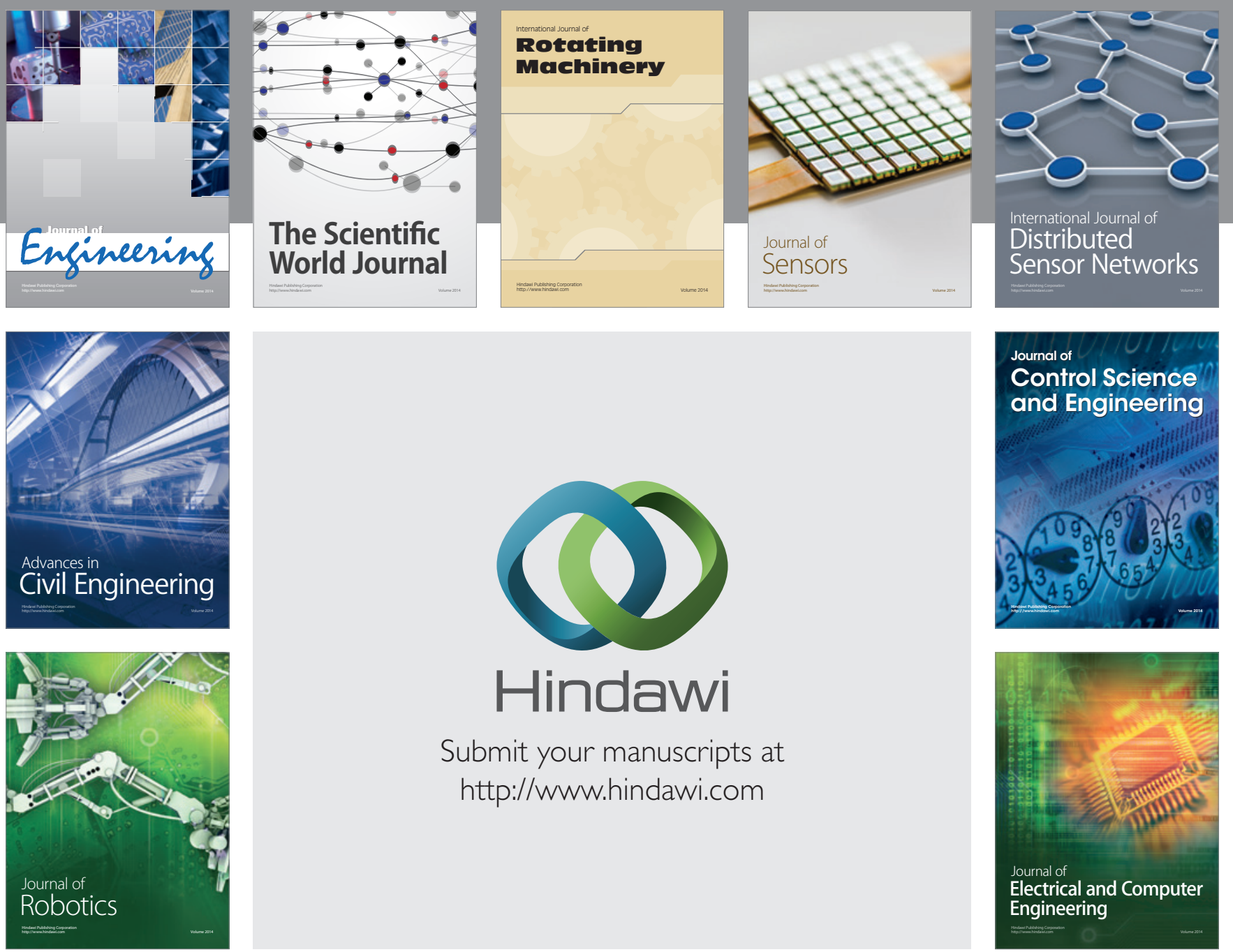

Submit your manuscripts at

http://www.hindawi.com
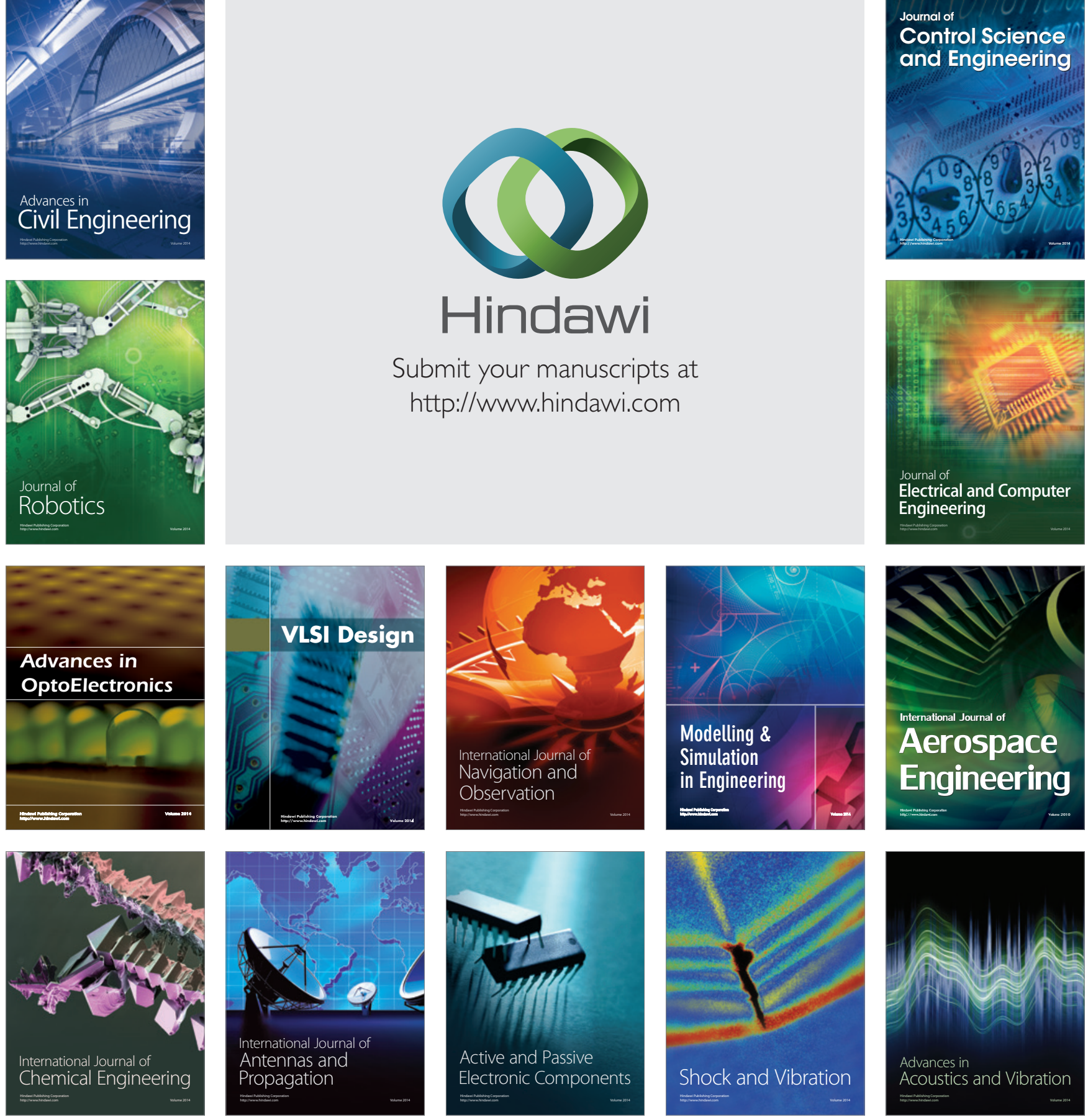